Article

\title{
Adverse drug reaction reports containing AMR-relevant MedDRA Terms in the Dutch Pharmacovigilance database
}

\author{
Jean Marie Vianney Habarugira, ${ }^{1,2 *}$, Linda Härmark ${ }^{3}$, Albert Figueras ${ }^{2}$ \\ 1 European \& Developing Countries Clinical Trials Partnership (EDCTP) \\ 2 Departament de Farmacologia, de Terapèutica i de Toxicologia. Universitat Autònoma de Barcelona (UAB), \\ Barcelona, Spain \\ 3 Netherlands Pharmacovigilance Centre Lareb, 's-Hertogenbosch, The Netherlands \\ Corresponding author: Habarugira@edctp.org
}

ORCID IDs :

JMV Habarugira: 0000-0003-1267-5972

Linda Härmark: 0000-0002-9314-9298

A Figueras: 0000-0002-2740-2013

\begin{abstract}
(1) Background: Antimicrobial resistance (AMR) requires urgent multidisciplinary solutions, and Pharmacovigilance (PV) has the potential to strengthen current antimicrobial stewardship (AMS) strategies. This study aimed to characterise AMR-relevant adverse drug reaction (ADR) reports submitted to The Netherlands Pharmacovigilance Centre (Lareb); (2) Methods: We carried out a descriptive analysis of ADR reports submitted to Lareb, coded with AMR-relevant MedDRA Preferred Terms (PTs).; (3) Results: Between 1998 and Jan 2019, 252 AMR-relevant ADR reports were submitted to Lareb. The most frequent antibiotics were tobramycin $(n=89 ; 35 \%)$, colistin $(n=30 ; 11,9 \%)$, ciprofloxacin $(n=16 ; 6,3 \%)$, doxycycline $(n=14 ; 5,5 \%)$ and aztreonam $(n=12 ; 4,8 \%)$. The PTs used included Off label use $(n=91 ; 36,1 \%)$, drug ineffective $(n=71 ; 28,2 \%)$, product use in unapproved indication $(n=28 ; 11,1 \%)$, pathogen resistance $(n=14 ; 5,6 \%)$ and drug resistance $(n=13$; $5,2 \%) .54 \%$ of the reports were on Watch antibiotics and $19 \%$ involved the Reserve group. In the Watch group, "Off label use" and "Product use in unapproved indication" were the most frequent PTs and majority of reports on Reserve antibiotics were coded as "Off label". (4) Conclusions: Addressing AMR using the PV methods will provide an opportunity for PV expansion, especially in resource-limited settings, encouraging further investments into AMS programs and PV systems.
\end{abstract}

Keywords: antibiotics, antimicrobial resistance, antimicrobial stewardship, AWaRe, Pharmacovigilance, Lareb, adverse drug reactions,

\section{Introduction}

\section{Antimicrobial resistance (AMR) and antimicrobial stewardship (AMS)}

Antimicrobials include a wide category of medicines used to prevent and treat infections in humans, animals, and plants [1]. These medicines are designed to kill or inhibit growth of microorganisms responsible for infections, but with time, antimicrobial resistance (AMR) occurs as the same microorganisms develop the ability to resist to the antimicrobial action of medicines previously effective [2]. Antimicrobial Stewardship (AMS) designates the set of programmes and actions conducted at different levels of the value chain to encourage the responsible use of antimicrobials through the delivery of multiple evidence-based interventions with the final aim of ensuring an optimal selection of the antibiotic when it is needed, and its prescription at the required dosage and duration of treatment resulting in the best clinical outcome, with minimized toxicity to the patient 
and minimum impact on subsequent resistance $[3,4]$. Whilst measures have been put in place to address AMR at hospital or country level, there is still lack of a globally coordinated strategy to curb the increasing resistance [5], especially because less AMS interventions cover primary health care, the dispensing of antimicrobials without prescription in pharmacies, as well as the extended use of antimicrobials in non-human sectors [6]. As a program, AMS is a set of all interventions used to enhance the rational use of antibiotics [7]. Various forms of antimicrobial stewardship programs have been established in different countries [8] at different levels of care delivery with involvement of a wide range of stakeholders including clinicians, pharmacists, nurses, and administrators and healthcare facilities $[9,10,11]$. As the world puts in place various measures to curb the rising threat caused by the rising resistance to existing antibiotics, Pharmacovigilance methods could constitute an important part of the wider multi-disciplinary approaches used for resistance surveillance and warning.

\section{Pharmacovigilance and Antimicrobial Resistance}

Pharmacovigilance (PV) is the science and activities relating to the detection, assessment, understanding and prevention of adverse effects or any other possible drug-related problems [12]. Adverse effects and other drugrelated problems include a range of effects that follow use of a medicinal product with varying degree of harm and causality certainty. An adverse effect of a medicinal product is a negative or harmful patient outcome that seems to be associated with the treatment, including there being no effect at all. An adverse drug reaction (ADR) is referred to when causality analysis has taken place and the link between the medicine and a suspected adverse effect is beyond uncertainty [13]. In the context of this study, the terms adverse drug reaction (ADR) reports refer to reports sent by health professionals or patients when an adverse effect has occurred in a patient taking one or more antimicrobial. The scientific community continues to propose innovative approaches, and some have suggested pharmacovigilance databases and methods as a potential tool to consider for the setup of antimicrobial stewardship [14]. Recent studies have underlined the potential role of Pharmacovigilance in containing the rising antimicrobial resistance $[15,16]$ proposing methods and tools that PV can offer to programs that monitor suspected resistance or cases of inappropriate use of antimicrobials. A recent study conducted in Russia concluded that the most frequent types of medication errors associated with the use of beta-lactams were the leading risk factors of growing bacterial resistance [17], and the authors emphasized on the need to have proper code to report medication error events involving antibiotics. Other researchers have looked at therapeutic failure as a reportable event but recognised the need to use the right definition of failure [18] if pharmacovigilance systems are to systematically collect data on failure. Researchers have also emphasized on the importance of PV databases which constitute a unique resource of information on potential misuse of medicines, including antimicrobials, and potentially containing AMR-relevant data [19]. A recent study has identified a set of 17 MedDRA Preferred Terms which suggest suspicion of Resistance, Ineffectiveness, Off-label use and medication errors [20].

\section{Pharmacovigilance in The Netherlands}

Established in 1968, the WHO Programme for International Drug Monitoring (PIDM) had The Netherlands as one its ten founding members. Since then, the program has expanded to include 145 full member countries and 26 associate member countries [21]. The Netherlands Pharmacovigilance Center Lareb manages a spontaneous reporting system, which involves collection and evaluation of suspected adverse drug reactions (ADRs) of medicinal products, aiming to identify new safety signals. Lareb codes the reports using MedDRA, and the reports are assessed by qualified assessors before entry into the database and prior to sharing with the global community via PIDM. To determine if specific reports can be qualified for signal review, a rigorous scientific review must take place, and signals are shared with the Dutch Medicines Evaluation Board (CBG/MEB) which will decide, often in the European context, if further regulatory action is necessary [22]. As a founding member of the IPDM, The Netherlands has a rich experience in ADR reports collection and sharing with the world through the UMC database. The Netherlands Pharmacovigilance Centre (Lareb) was designated in 2013 as the WHO collaborating centre for patient reporting [23] and the strength of the centre lies among other things, in the collection of reports directly via the patient reporting scheme initiated in 2003 and went on to become a reliable of source of safe data $[24,25]$.

\section{The Access, Watch and Reserve (AWaRe) classification for availability and appropriate use}

Antibiotic resistance affects people of all ages in all countries. Yearly, an estimated 5.7 million deaths occur from treatable infectious diseases, mostly in low- and middle-income countries (LMICs), and many of these lives could have be saved if effective antibiotics were available. At the same time, there are about 700,000 annual deaths worldwide due to antibiotic resistance. The development of resistance, while threatening the right to best medical care, has another side of the coin showing us that resource-limited settings are more affected by lack of access to antibiotics than by the resistance. Preserving antibiotic effectiveness while ensuring universal access is at the heart of public health dilemmas, as policies for good access must be accompanied with strong measures to 
minimise inappropriate use that would lead to further resistance [26, 27]. To address the issue of availability while ensuring appropriate use, since 2017, the Word Heal Organisation (WHO) Essential Medicines List (EML) includes a classification of antimicrobials into three categories known as "Access", "Watch" and "Reserve" (AWaRe), based on the indication, availability, and awareness [28]. A global campaign was launched in 2019 urging governments to implement to implement the AWaRe tool through national guidelines to reduce antimicrobial resistance and ensure access [29].

\section{Study objective}

In a previous study [20], a list seventeen of MedDRA Preferred Terms relevant to AMR or inappropriate use of antimicrobials was established based on global data from VigiBase. Focusing on country-specific data, the objective of this research was to characterise ADR reports submitted to The Netherlands Pharmacovigilance Centre (Lareb) following use of antibiotics and coded with MedDRA Preferred Terms that suggest suspicion of Resistance, Ineffectiveness, Off-label use or medication Errors.

\section{Material and Methods}

\section{Data source and Search strategy}

We carried out a descriptive analysis of ADR reports submitted to The Netherlands Pharmacovigilance Center (Lareb) database, fulfilling the following criteria:

a) Reports on Antibiotics classified under ATC J01 or ATC J04.

b) Reports coded with at least one of the following MedDRA (version 21.1) Preferred Terms and codes: Pathogen resistance (10034133); Drug ineffective (10013709); Treatment failure (10066901); Drug resistance (10059866); Therapeutic product ineffective (10060769); Therapy non-responder (10051082); Decreased activity (10011953); Drug ineffective for unapproved indication (10051118); Therapeutic response decreased (10043414); Multiple drug resistance (10048723); Off label use (10053762); Medication error (10027091); Product use in unapproved indication (10076476); Contraindicated product administered (10078504)

For each report meeting the criteria in a) and b), the following information was collected and included in the dataset for further analysis: Report identifier; Suspected ADR; Year of report; Reporter type; Suspected drug; Indication and Action taken

\section{Results}

\subsection{ADR reports with AMR-relevant codes}

Between 1998 and January 2019, a total of 252 ADR reports (study sample) were submitted to Lareb using a PT or a combination of PTs that suggested suspicion of AMR or use-related issues (irrational use or medication errors). The following antibiotics were the most frequently reported as suspected causes of AMR-relevant ADRs: tobramycin $(n=89 ; 35 \%)$, colistin $(n=30 ; 11,9 \%)$, ciprofloxacin $(n=16 ; 6,3 \%)$, doxycycline $(n=14 ; 5,5 \%)$ and aztreonam $(\mathrm{n}=12 ; 4,8 \%)$. The most frequently used PTs were off label use $(\mathrm{n}=91 ; 36,1 \%)$, drug ineffective $(\mathrm{n}=71$; $28,2 \%)$, product use in unapproved indication $(n=28 ; 11,1 \%)$, pathogen resistance $(n=14 ; 5,6 \%)$ and drug resistance $(n=13$; $5,2 \%)$

\subsection{Most frequently used PTs in cases of suspected resistance or use-related issues}

As shown in Table 1, 98 reports (39\% of the study sample) suggested suspicion of resistance using PTs such as drug ineffective, pathogen resistance and drug resistance. A larger group of reports $(\mathrm{n}=119 ; 47 \%$ of the study sample) included PTs suggesting use-related issues such as off label use $(\mathrm{n}=91 ; 76 \%)$ and product use in unapproved indication $(28 ; 15 \%)$. More than half of the 91 reports coded with PT off label use described events in patients on tobramycin as the suspect drug ( $n=53 ; 58 \%)$; additionally, 24 (26\%) were on colistin. The reports coded with the PT product use in unapproved indication were predominantly on tobramycin ( $\mathrm{n}=27 ; 96 \%$ of the cases). Out of the 252 reports of the study sample, 35 (14\%) were coded each with more than 1 PT, combining PTs that refer to suspicion of both resistance and use-related issues

\subsection{Applying the AWaRe Classification to the reports}

As shown in Table 2, the Watch category was involved in 137 (54\%) of the 252 ADR reports with a predominance of tobramycin with 89 of the 147 Watch reports $(n=89 ; 78 \%)$. The second leading group is Reserve with 45 reports (19\%), followed by the Access group with 40 reports (16\%), and the remaining $11 \%$ include combination of antibiotics from different AWaRe groups or non-AWaRe classified antibiotics. In the Watch group, "Off label use" and "Product use in unapproved indication" were the most frequent PTs, used in 57 (42\%) and 27 (20\%) 
reports, respectively. Majority ( 25 or $76 \%$ ) of reports involving Reserve antibiotics were submitted as Off label. In the Access group, "drug ineffective" was the most frequent PT in 25 (63\%) out of 40 reports.

\section{3. "Off label use" and "product use in an unapproved indication": tobramycin and colistin}

In the current study we found that out of the 252 ADR reports, $119(47 \%)$ are suggesting use-related issues. Of these 119 reports, 91(76\%) are coded as "off label use" and the remaining 28 (15\%) reports are coded "product use in an unapproved indication". The 91 off label ADR reports include predominantly tobramycin (Watch) and colistin (Reserve) as the suspected drug with 53 (58\%) and 24 (26\%) reports, respectively. A focus on 89 ADR reports with tobramycin as the suspected drug, 30 (33.7\%) reports included chronic obstructive pulmonary disease as the primary indication while $25(28 \%)$ reports had bronchiectasis as the indication. 53 of the 89 ADR reports were coded as off label use in treating chronic obstructive pulmonary disease (22 cases) and bronchiectasis (13 cases).

\subsection{The 2015 peak in numbers of AMR-relevant ADR reports to Lareb}

From the 252 ADR reports submitted over a period of about 20 years, 82 (34\%) reports were submitted in 2015 as illustrated by Figure 1. A sharp increase in the number of reports was observed in the three consecutive years with 21 in 2013, 54 in 2014 and 83 in 2015. The numbers dropped to 26 reports in 2016, but in just these four years, Lareb received $73 \%$ (183 of 252) of AMR-relevant reports received over a period of 20 years. In this short period of sharp increase, there was a clear increase of tobramycin reports passing from 11 in 2013 to 30 in 2014 and reaching 39 in 2015. Colistin shows a visible increase of ADR reports in this period, passing from 0 in 2013 to 10 in 2014 and 14 in 2015. Other drugs with increasing ADR reports numbers in the spike period include aztreonam and doxycycline. Of the 183 reports submitted in the period 2013-2016, 84 reports were on suspected "off label use" with a predominance of tobramycin that was the suspect drug in 50 (of 84) reports. 39 of the 183 reports were submitted with the PT "drug ineffective" and diverse antibiotics were reported as suspected drugs. However, with reports coded using the PT "product use in unapproved indication" indication, a clear predominance of tobramycin was observed.

Table 1 The most frequently used PTs and reported antibiotics per RIOLE group

\begin{tabular}{|c|c|c|}
\hline AMR-relevant PT & PT $(\mathrm{n} ; \%)$ in the RIOLE category & Most reported antibiotics $(\mathrm{n} ; \%)$ \\
\hline \multirow{8}{*}{$\begin{array}{l}\text { Suggesting AMR } \\
(98 ; 39 \%)\end{array}$} & \multirow{3}{*}{ drug ineffective $(71 ; 72 \%)$} & aztreonam $(9 ; 13 \%)$ \\
\hline & & amoxicillin+Beta-lactamase inhibitor $(6 ; 8 \%)$ \\
\hline & & doxycycline $(6 ; 8 \%)$ \\
\hline & \multirow[t]{3}{*}{ pathogen resistance $(14 ; 14 \%)$} & ceftazidime $(5 ; 36 \%)$ \\
\hline & & ciprofloxacin $(2 ; 14 \%)$ \\
\hline & & linezolid $(2 ; 14 \%)$ \\
\hline & \multirow[t]{2}{*}{ drug resistance $(13 ; 13 \%)$} & tobramycin $(3 ; 23 \%)$ \\
\hline & & ciprofloxacin $(2 ; 15 \%)$ \\
\hline \multirow{4}{*}{$\begin{array}{l}\text { Suggesting use-related issues } \\
(119 ; 47 \%)\end{array}$} & \multirow[t]{3}{*}{ off-label use $(91 ; 76 \%)$} & tobramycin $(53 ; 58 \%)$ \\
\hline & & colistin $(24 ; 26 \%)$ \\
\hline & & doxycycline $(6 ; 7 \%)$ \\
\hline & $\begin{array}{l}\text { product use in unapproved } \\
\text { indication }(28 ; 15 \%)\end{array}$ & tobramycin $(27 ; 96 \%)$ \\
\hline $\begin{array}{l}\text { Suggesting both AMR and use- } \\
\text { related issues }(35 ; 14 \%)\end{array}$ & $\begin{array}{l}\text { Combinations of PTs } \\
\text { Suggesting both AMR and use- } \\
\text { related issues }(35 ; 14 \%)\end{array}$ & $\begin{array}{l}\text { ciprofloxacin }(7 ; 20 \%) \\
\text { azithromycin }(3 ; 9 \%)\end{array}$ \\
\hline TOTAL $=252 ; 100 \%$ ADR reports & & - \\
\hline
\end{tabular}


Table 2 The most reported antibiotics and used PTs per AWaRe class

\begin{tabular}{|c|c|c|}
\hline AWaRe categories & Most reported antibiotics (n; \%) & Most used PTs per AWaRE category $(n ; \%)^{*}$ \\
\hline \multirow[t]{3}{*}{ Access $(40 ; 16 \%)$} & doxycycline $(14 ; 35 \%)$ & \multirow{3}{*}{$\begin{array}{l}\text { drug ineffective }(25 ; 63 \%) \\
\text { off label use }(6 ; 15 \%)\end{array}$} \\
\hline & $\begin{array}{l}\text { amoxicillin+Beta-lactamase inhibitor } \\
(7 ; 18 \%)\end{array}$ & \\
\hline & sulfamethoxazole + trimethoprim $(4 ; 10 \%)$ & \\
\hline \multirow[t]{4}{*}{ Watch $(137 ; 54 \%)$} & tobramycin $(89 ; 78 \%)$ & \multirow{4}{*}{$\begin{array}{l}\text { Off label use }(57 ; 42 \%) \\
\text { Product use in unapproved indication }(27 ; 20 \%) \\
\text { drug ineffective }(20 ; 15 \%) \\
\text { pathogen resistance }(8 ; 6 \%)\end{array}$} \\
\hline & ciprofloxacin $(16 ; 33 \%)$ & \\
\hline & azithromycin $(8 ; 17 \%)$ & \\
\hline & moxifloxacin $(7 ; 15 \%)$ & \\
\hline \multirow[t]{2}{*}{ Reserve $(45 ; 19 \%)$} & colistin $(30 ; 91 \%)$ & \multirow[t]{2}{*}{ off label use $(25 ; 76 \%)$} \\
\hline & aztreonam $(12 ; 11 \%)$ & \\
\hline $\begin{array}{l}\text { Combination of different } \\
\text { classes }(17 ; 6 \%)\end{array}$ & concomitant from different classes & $\begin{array}{l}\text { drug ineffective }(7 ; 41 \%) \\
\text { drug resistance }(3 ; 18 \%)\end{array}$ \\
\hline $\begin{array}{l}\text { Other or not classified (13; } \\
5 \%)\end{array}$ & & drug ineffective $(8 ; \%)$ \\
\hline TOTAL (252;100\%) & - & - \\
\hline
\end{tabular}

* Most frequently used PT per AWaRe class (access, watch, reserve) 
Figure 1 Yearly reports from 1998 to Jan 2019

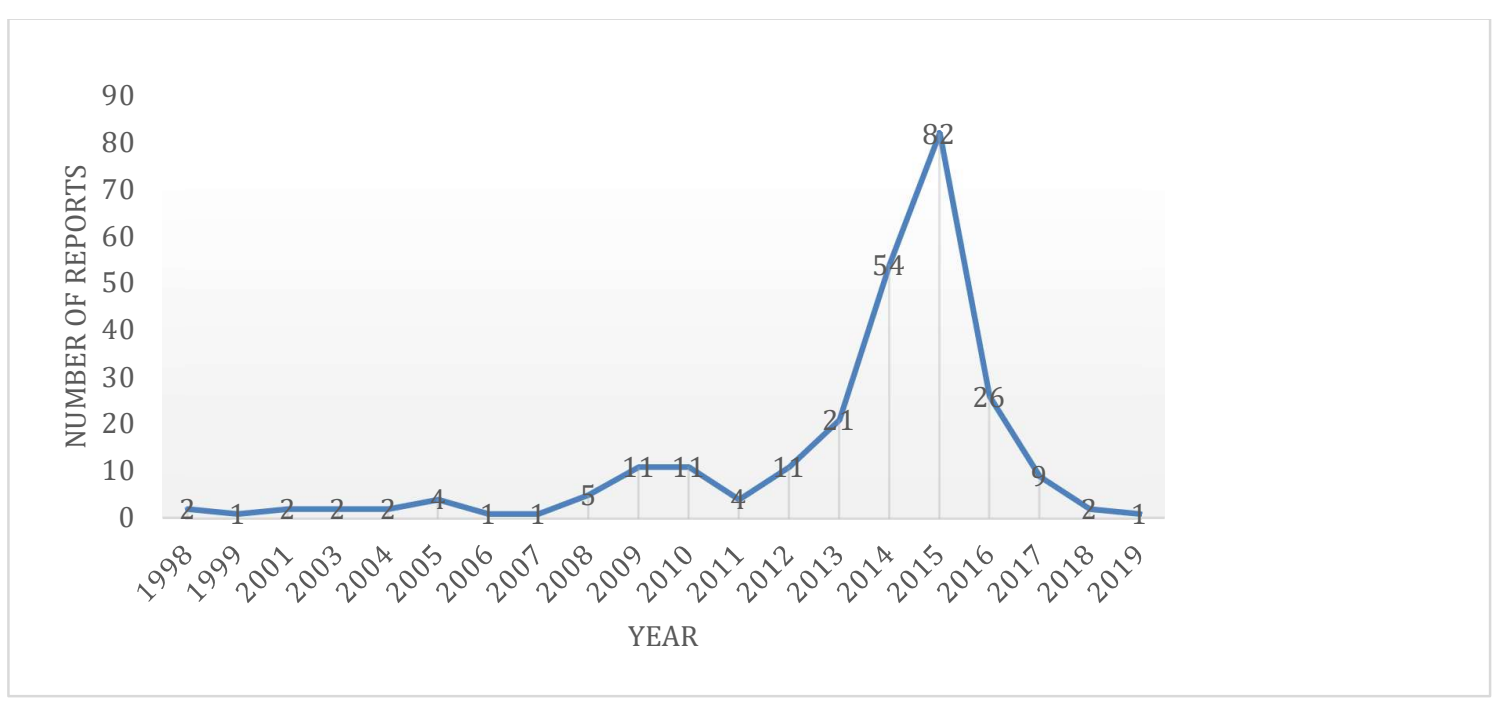

\section{Discussion and Conclusion}

As the world seeks and puts in place strategies to tackle the rising AMR, living reviews of Pharmacovigilance data should be seen as a strong potential source of data on trends in suspected resistance and possible irrational use of antimicrobials, especially in countries with less resources and few testing laboratories. This study revealed an increase in AMR-relevant reports with a peak in 2015, and tobramycin appeared to be involved in majority of reports. Such a cluster in time and maybe geographically localisable could be used by AMS programs to trigger a drug-focussed monitoring, e.g. tobramycin in this case. The present analysis of the Lareb database, a consolidated pharmacovigilance program has shown that this use of PV databases to investigate AMR issues is possible. Even more, despite the phenomenon of underreporting (common to most PV programmes based on spontaneous reporting) and the lack of proactive promotion of these preferred terms in the PV dictionaries, 252 reports were identified. Furthermore, $19 \%$ of these reports involved a Reserve antibiotic and 54\% a Watch antibiotic, both belonging to categories of products of special interest from the AMR perspective.

Systematic collection of data on use-related issues should be used to provide a bigger picture to prescribers who have a choice to make during each consultation. Reports on off label use of drug use in contraindicated indications should be shared with those in the antimicrobials supply and use chain to timely inform their decision-making process.

The 91 Off label ADR reports included tobramycin (Watch) and colistin (Reserve) as the suspected drug with 53 $(58 \%)$ and $24(26 \%)$ reports, respectively. Special attention should be given to these groups, and AMS programs can make important steps in the right direction if all involved parties

Tobramycin is an aminoglycoside used to treat several conditions, including superficial and deep infections $[\mathrm{xxx}]$. Depending on the indication and route of administration, various forms of tobramycin are marketed, including inhaled tobramycin used to treat cystic fibrosis [xxxi], ophthalmic solution to treat bacterial conjunctivitis [xxxii], intramuscular injection and intravenous infusion for the treatment of other bacterial infections.

Less used as of the 1980s due to safety issues after more than 30 decades on the market, colistin, a polymyxin antibiotic, became later in the 21st century, a last-resort drug for multi-drug resistant Gram-negative organisms [xxxiii, xxxiv]. In this study, we found that this Reserve antimicrobial is reported as the suspected drug in 24 reports with ADRs coded as "off label use". The leading indications for which colistin was prescribed are chronic obstructive pulmonary disease and bronchiectasis

The antimicrobials supply and use chain, important stakeholders include marketing authorisation holders $(\mathrm{MAH})$, prescribers, pharmacists, and patients. It should be noted that in many countries, the function of 
pharmacist is supported by other distribution services such drug stores that provide over the counter (OTC) medicines. MAHs have the obligation to regularly submit safety reports to National competent authorities (NCAs). But, more than just an obligation pharmacists and other products distributors play an important role in collecting safety data and submitting high quality safety reports to competent authorities. In countries where OTC medicines include antibiotics, the supply chain must be considered in all AMS programs using local PV systems to collect AMR-relevant data.

In this study we have noted an increased number of reports in the period 2013-2015 and majority of these reports were collected and submitted by MAHs. Beside regulatory obligation, there should be a collaborative approach between MAHs and PV centers such as Lareb, to facilitate joint assessment of suspected cases if necessary. PV centers are part of a large global network sharing safety data through PIDM, and the AMR PV researchers can benefit from reports that have gone through causality assessment by both MAHs and PV centers.

In many countries, health care professionals play a crucial role in gathering and submitting safety data to competent authorities. MAHs rely on health care professionals working on clinical trials or seeing patients in clinics to provide solicited or unsolicited safety data on products. In their risk management plans (RMPs), MAHs are required to address undesirable clinical outcomes and for which there is sufficient scientific evidence that they are caused by the medicinal product [xxxv]. If the drug safety community agrees that resistance or ineffectiveness are undesirable clinical outcomes caused by the antimicrobial, the MAHs should consider the use of AMR-relevant MedDRA terms in collecting data to inform their RMPs. The Guideline on good pharmacovigilance practices (GVP) Module V (see V.B.5.9. RMP part II, module SVIII "Summary of the safety concerns") also indicates that reports of adverse reactions may be derived from multiple sources including spontaneous data sources and may be linked to situations such as off label use and medication errors. If the concerned medicinal product is antimicrobial, regulatory activities should consider the risk associated with off label use and medication in the antimicrobial stewardship context.

Expanding the role of PV requires both promotion of existing tools and education for the potential reports of observed or suspected effects. In this study, a large portion of analysed reports were collected and submitted by MAHs and there may be other potential similar cases that went unreported by prescribers and patients.

Promoting the existence of AMR-relevant MedDRA terms and explaining their relevance to surveillance of antimicrobial resistance could lead to increased submission of similar reports from prescribers and patients. Public health will gain from different angles if PV is integrated in the AMS programmes package. PV centers at country level should be encouraged to actively promote these AMR relevant PTs and invite reporters to collect and send this information to relevant agencies. By getting actively involved in this process, (1) PV centres will receive more spontaneous reports, the centres work will be more visible and appreciated by other public health stakeholders, (2) suspected AMR could be timely detected, potential clusters could be highlighted and, if necessary, ad-hoc microbiological tests could be conducted. The integration of PV in this process could lead to a win-win situation for different scientific disciplines tackling AMR from traditionally isolated perspectives.

Pharmacovigilance is already a multidimensional science with potential to expand further. Tackling AMR questions using the existing PV methods will also provide an opportunity for PV as a field to evolve further and countries will see more value in investing in their PV systems. As technology drives further the field of Pharmacovigilance, methods traditionally used in post-marketing drug safety monitoring have the potential to serve as surveillance strategies for Antimicrobial Stewardship programs.

Conflicts of Interest: The authors declare no conflict of interest.

\section{References}

[1] Antimicrobial Resistance.https://www.who.int/news-room/fact-sheets/detail/antimicrobial-resistance. Accessed 4 Jul 2021

[2] About antibiotic resistance. https://www.cdc.gov/drugresistance/about.html Accessed 4 Jul 2021

[3] Ashiru-Oredope D, Doble A, Akpan MR, Hansraj S, Shebl NA, Ahmad R, Hopkins S. Antimicrobial Stewardship Programmes in Community Healthcare Organisations in England: A Cross-Sectional Survey to Assess Implementation of Programmes and National Toolkits. Antibiotics (Basel). 2018 Nov 7;7(4):97. doi: 10.3390/antibiotics7040097. PMID: 30405004; PMCID: PMC6316239. 
[4] Doron, S., \& Davidson, L. E. (2011). Antimicrobial stewardship. Mayo Clinic proceedings, 86(11), 11131123. https://doi.org/10.4065/mcp.2011.0358

[5] Hamdan, S. and El-Dahiyat, F. (2020), Implementation and evaluation of an antimicrobial stewardship program across nine hospitals in the United Arab Emirates: a qualitative study. J Pharm Pract Res, 50: 124-131. https://doi.org/10.1002/jppr.1613

[6] B. onas, Olga B.; Irwin, Alec; Berthe,Franck Cesar Jean; Le Gall,Francois G.; Marquez,Patricio V..2017. Drug-resistant infections: a threat to our economic future (Vol. 2) : final report (English). HNP/Agriculture Global timicrobial Resistance Initiative Washington, D.C.: World Bank Group. http://documents.worldbank.org/curated/en/323311493396993758/final-report

[7] Shirazi OU, Ab Rahman NS, Zin CS. A Narrative Review of Antimicrobial Stewardship Interventions within In-patient Settings and Resultant Patient Outcomes. J Pharm Bioallied Sci. 2020;12(4):369-380. doi:10.4103/jpbs.JPBS_311_19

[8] May L, Martín Quirós A, Ten Oever J, Hoogerwerf J, Schoffelen T, Schouten J. Antimicrobial stewardship in the emergency department: characteristics and evidence for effectiveness of interventions. Clin Microbiol Infect. 2021 Feb;27(2):204-209. doi: 10.1016/j.cmi.2020.10.028. Epub 2020 Nov 2. PMID: 33144202.

[9] Acquisto NM, May L. Collaborative Antimicrobial Stewardship in the Emergency Department. Infect Dis Clin North Am. 2020 Mar;34(1):109-127. doi: 10.1016/j.idc.2019.10.004. PMID: 32008694.

[10] Buckel WR, Kaye KS, Patel PK. Collaborative Antimicrobial Stewardship: Working with Hospital and Health System Administration. Infect Dis Clin North Am. 2020 Mar;34(1):1-15. doi: 10.1016/j.idc.2019.10.003. Epub 2019 Dec 10. PMID: 31836330.

[11] Olans RD, Hausman NB, Olans RN. Nurses and Antimicrobial Stewardship: Past, Present, and Future. Infect Dis Clin North Am. 2020 Mar;34(1):67-82. doi: 10.1016/j.idc.2019.10.008. PMID: 32008696.

[12] Pharmacovigilance: Overview I European Medicines Agency (europa.eu) Available at https://www.ema.europa.eu/en/human-regulatory/overview/pharmacovigilance-overview Accessed 13 Jun 2021

[13] UMC. Glossary of pharmacovigilance terms https://www.who-umc.org/globalpharmacovigilance/publications/glossary/. Accessed 13 Jul 2021

[14] Antimicrobial Resistance - An overlooked Adverse event. https://www.whoumc.org/media/2775/web uppsalareports issue74.pdf Accessed 13 Jun 2021

[15] Agrawal V, Shrivastava TP, Adusumilli PK, Vivekanandan K, Thota P, Bhushan S. Pivotal role of Pharmacovigilance Programme of India in containment of antimicrobial resistance in India. Perspect Clin Res. 2019;10(3):140-144. doi:10.4103/picr.PICR_29_18

[16] Laxminarayana Kurady Bairy, Veena Nayak, Avinash A \& Sushil Kiran Kunder (2016) Advances in pharmacovigilance initiatives surrounding antimicrobial resistance-Indian perspective, Expert Opinion on Drug Safety, 15:8, 1055-1062, DOI: 10.1080/14740338.2016.1182495

[17] Kuzmina, A.V., Asetskaya, I.L., Zyryanov, S.K. et al. Detecting medication errors associated with the use of beta-lactams in the Russian Pharmacovigilance database. BMC Pharmacol Toxicol 22, 5 (2021). https://doi.org/10.1186/s40360-020-00470-x

[18] Ruíz-Garzón, J. A., \& Calderón-Ospina, C. A. (2019). Consideraciones acerca del reporte y la evaluación del fallo terapéutico en farmacovigilancia/Considerations regarding the reporting and evaluation of therapeutic failure in pharmacovigilance. Revista De La Facultad De Medicina, 67(3), 287-292. doi:http://dx.doi.org.are.uab.cat/10.15446/revfacmed.v67n3.65801

[19] Habarugira, J.M.V., Figueras, A. Antimicrobial stewardship: can we add pharmacovigilance networks to the toolbox? Eur J Clin Pharmacol 77, 787-790 (2021). https://doi.org/10.1007/s00228-020-03035-3

[20] Habarugira, JMV, Figueras, A. Pharmacovigilance network as an additional tool for the surveillance of antimicrobial resistance. Pharmacoepidemiol Drug Saf. 2021; 1-9. https://doi.org/10.1002/pds.5249

[21] Members of the WHO Programme for International Drug Monitoring. Available at https://www.whoumc.org/global-pharmacovigilance/who-programme-for-international-drug-monitoring/whoprogramme-members/ Accessed 13 Jun 2021

[22] Härmark, L., van Hunsel, F. \& Grundmark, B. ADR Reporting by the General Public: Lessons Learnt from the Dutch and Swedish Systems. Drug Saf 38, 337-347 (2015). https://doi.org/10.1007/s40264-0150264-1

[23] Florence van Hunsel, Linda Härmark \& Leàn Rolfes (2019) Fifteen years of patient reporting -what have we learned and where are we heading to?, Expert Opinion on Drug Safety, 18:6, 477484, DOI: $\underline{10.1080 / 14740338.2019 .1613373}$ 
[24] van Grootheest AC, Passier JL, van Puijenbroek EP. Meldingen van bijwerkingen rechtstreeks door patiënten: gunstige ervaringen van het eerste jaar [Direct reporting of side effects by the patient: favourable experience in the first year]. Ned Tijdschr Geneeskd. 2005 Mar 5;149(10):529-33. Dutch. PMID: 15782689.

[25] de Langen J, van Hunsel F, Passier A, de Jong-van den Berg L, van Grootheest K. Adverse drug reaction reporting by patients in the Netherlands: three years of experience. Drug Saf. 2008;31(6):515-24. doi: 10.2165/00002018-200831060-00006. PMID: 18484785.

[26] Stig Wall (2019) Prevention of antibiotic resistance - an epidemiological scoping review to identify research categories and knowledge gaps, Global Health Action, 12:sup1, DOI: 10.1080/16549716.2020.1756191

[27] New report calls for urgent action to avert antimicrobial resistance crisis. https://www.who.int/news/item/29-04-2019-new-report-calls-for-urgent-action-to-avert-antimicrobialresistance-crisis. Accessed 21 Jul 2021

[28] Sharland M, Pulcini C, Harbarth S, et al. Classifying antibiotics in the WHO Essential Medicines List for optimal use-be AWaRe. The Lancet Infectious Diseases. 2018;18(1):18-20. http://doi.org/10.1016/s14733099(17)30724-7.

[29] AWaRe Policy Brief. https://adoptaware.org/assets/pdf/aware_policy brief.pdf Accessed 18 Jul 2021

[30] Reyhanoglu G, Reddivari AKR. Tobramycin. [Updated 2021 Jun 10]. In: StatPearls [Internet]. Treasure Island (FL): StatPearls Publishing; 2021 Jan-. Available from: https://www.ncbi.nlm.nih.gov/books/NBK551695/

[31] Hagerman JK, Knechtel SA, Klepser ME. Tobramycin solution for inhalation in cystic fibrosis patients: a review of the literature. Expert Opin Pharmacother. 2007 Mar;8(4):467-75. doi: 10.1517/14656566.8.4.467. PMID: 17309341.

[32] Kernt K, Martinez MA, Bertin D, Stroman D, Cupp G, Martínez C, Tirado M, Guasch J; International Tobrex $2 x$ Group (Eu). A clinical comparison of two formulations of tobramycin $0.3 \%$ eyedrops in the treatment of acute bacterial conjunctivitis. Eur J Ophthalmol. 2005 Sep-Oct;15(5):541-9. PMID: 16167284.

[33] Dhariwal AK, Tullu MS. Colistin: re-emergence of the 'forgotten' antimicrobial agent. J Postgrad Med. 2013 Jul-Sep;59(3):208-15. doi: 10.4103/0022-3859.118040. PMID: 24029199.

[34] Molina J, Cordero E, Pachón J. New information about the polymyxin/colistin class of antibiotics. Expert Opin Pharmacother. 2009 Dec;10(17):2811-28. doi: 10.1517/14656560903334185. PMID: 19929704.

[35] Guideline on good pharmacovigilance practices (GVP) Module V - Risk management systems (Rev 2) (europa.eu) - https://www.ema.europa.eu/en/documents/scientific-guideline/guideline-goodpharmacovigilance-practices-module-v-risk-management-systems-rev-2_en.pdf 21 Jul 2021 